\title{
ON THE 2-ORTHOGONAL POLYNOMIALS AND THE GENERALIZED BIRTH AND DEATH PROCESSES
}

ZEROUKI EBTISSEM AND BOUKHEMIS AMMAR

Received 16 May 2005; Revised 1 May 2006; Accepted 4 May 2006

We discuss the connections between the 2-orthogonal polynomials and the generalized birth and death processes. Afterwards, we find the sufficient conditions to give an integral representation of the transition probabilities from these processes.

Copyright (c) 2006 Hindawi Publishing Corporation. All rights reserved.

\section{Introduction}

The birth and death processes are closely related to the orthogonal polynomials. The latter allows determining the stochastic matrix associated with these processes.

Let us also note that these processes are stationary Markov processes whose state space is the nonnegative integers.

Many authors treated the question of the existing relationship between the birth and death processes and the orthogonal polynomials, in particular, in the works of Karlin and McGregor [6] and Ismail et al. [5].

The properties of these processes and of the orthogonal polynomials were the subject of other works, we can quote by the way of example Ismail et al. [3, 4], Maki [8], and Letessier and Valent [7].

In this paper, we will consider not only the orthogonal polynomials, but the 2-orthogonal polynomials and we will try to establish a bond between the latter and certain birth and death processes that will be called "generalized." These processes will have like transition probabilities

$$
P_{i j}(t)=\operatorname{Pr}\{X(t+s)=j / X(s)=i\}=\operatorname{Pr}\{X(t)=j, X(0)=i\}
$$

which satisfy, when $h \rightarrow 0$,

$$
P_{i j}(t)= \begin{cases}\lambda_{i} h+o(h), & j=i+1, \\ 1-\left(\lambda_{i}+\mu_{i}+\bar{\mu}_{i}\right) h+o(h), & j=i, \\ \mu_{i} h+o(h), & j=i-1, \\ \bar{\mu}_{i} h+o(h), & j=i-2, \\ o(h), & j \neq i-2, i-1, i, i+1,\end{cases}
$$

Hindawi Publishing Corporation

International Journal of Mathematics and Mathematical Sciences

Volume 2006, Article ID 28131, Pages 1-12

DOI 10.1155/IJMMS/2006/28131 
2 2-orthogonal polynomials and birth and death processes

$$
P_{i j}(0)=\delta_{i j}, \quad \forall i, j \in \mathbb{N},
$$

where $\lambda_{i}$ are the birth rates and $\mu_{i}$ and $\bar{\mu}_{i}$ are the death rates. It is also assumed that

$$
\lambda_{i}>0, \quad \mu_{i} \geq 0, \quad \bar{\mu}_{i+1}>0 ; \quad i \geq 0, \quad \bar{\mu}_{0} \geq 0 .
$$

These processes being stationary, $P_{i j}(t)$ does not depend on the way taken by the system to reach the state $j$, but depends only on the states $i$ and $j$ and of the laps of time $t$ taken while going from state $i$ towards state $j$. This is equivalent to

$$
\mathscr{P}(s+t)=\mathscr{P}(s) \mathscr{P}(t)
$$

In this work, we start initially by giving some properties of the "generalized" birth and death processes by determining the sequence of 2-orthogonal polynomials associated with this type of processes. Then, the sufficient conditions are given, which allow giving an integral representation of the transition probabilities from these processes.

We can quote as an example that this type of processes can be the modelling of problems met while studying the kinetics of enzymes, in particular, those which catalyze reactions to a substrate in the presence of noncompetitive inhibitors.

We will treat this type of model in future, when we study the generalized linear processes.

\section{The generalized Chapman-Kolmogorov equations}

Proposition 2.1. Let be a "generalized" birth and death process, where the transition probabilities are given by (1.2), then these probabilities satisfy two systems of differential recurrence relations called "Chapman-Kolmogorov (or C-K) equations." The forward C-K equations are

$$
\begin{aligned}
\frac{d}{d t} P_{i j}(t)= & \bar{\mu}_{j+2} P_{i, j+2}(t)+\mu_{j+1} P_{i, j+1}(t)+\lambda_{j-1} P_{i, j-1}(t), \\
& -\left(\bar{\mu}_{j}+\mu_{j}+\lambda_{j}\right) P_{i j}(t) .
\end{aligned}
$$

The backward C-K equations are

$$
\begin{aligned}
\frac{d}{d t} P_{i j}(t)= & \lambda_{i} P_{i+1, j}(t)+\mu_{i} P_{i-1, j}(t)+\bar{\mu}_{i} P_{i-2, j}(t) \\
& -\left(\bar{\mu}_{i}+\mu_{i}+\lambda_{i}\right) P_{i j}(t) .
\end{aligned}
$$

Proof. Since the process is stationary, on one hand we can write

$$
P_{i j}(t+s)=\sum_{k \geq 0} P_{i k}(t) P_{k j}(s), \quad s \longrightarrow 0,
$$


it follows from (1.2) that

$$
\begin{aligned}
\frac{d}{d t} P_{i j}(t) & =\lim _{s \rightarrow 0} \frac{P_{i j}(t+s)-P_{i j}(t)}{s} \\
& =\lim _{s \rightarrow 0} \frac{\sum_{k \geq 0}\left(P_{i k}(t) P_{k j}(s)-P_{i j}(t)\right)}{s} \\
& =\lim _{s \rightarrow 0}\left[\bar{\mu}_{j+2} P_{i j+2}(t)+\mu_{j+1} P_{i j+1}(t)+\lambda_{j-1} P_{i j-1}(t)-\left(\lambda_{j}+\mu_{j}+\bar{\mu}_{j}\right) P_{i j}(t)+\frac{o(s)}{s}\right],
\end{aligned}
$$

then we have (2.1).

On the other hand, by applying the semigroup property, we have also

$$
P_{i j}(s+t)=\sum_{k \geq 0} P_{i k}(s) P_{k j}(t), \quad s \longrightarrow 0,
$$

then from (1.2), we have

$$
\begin{aligned}
\frac{d}{d t} P_{i j}(t) & =\lim _{s \rightarrow 0} \frac{P_{i j}(t+s)-P_{i j}(t)}{s} \\
& =\lim _{s \rightarrow 0}\left[\lambda_{i} P_{i+1 j}(t)+\mu_{i} P_{i-1 j}(t)+\bar{\mu}_{i} P_{i-2 j}(t)-\left(\lambda_{i}+\mu_{i}+\bar{\mu}_{i}\right) P_{i j}(t)+\frac{o(s)}{s}\right] .
\end{aligned}
$$

So, we get (2.2)

Definition 2.2. The matrix $\mathscr{A}=\left(a_{i j}\right)_{i, j \in \mathbb{N}}$ defined by

$$
a_{i j}= \begin{cases}\lambda_{i} & \text { if } j=i+1, \\ \mu_{i} & \text { if } j=i-1, \\ \bar{\mu}_{i} & \text { if } j=i-2, \\ -\left(\lambda_{i}+\mu_{i}+\bar{\mu}_{i}\right) & \text { if } j=i, \\ 0 & \text { otherwise, }\end{cases}
$$

is called the infinitesimal generator of the process.

Lemma 2.3. A generalized birth and death process has the following properties:

(i) $\mathscr{P}^{\prime}(t)=\mathscr{P}(t) \mathscr{A}$,

(ii) $\mathscr{P}^{\prime}(t)=\mathscr{A} \mathscr{P}(t)$,

(iii) $\mathscr{P}(0)=I=\left(\delta_{i j}\right)_{i, j \in I N}$,

(iv) $P_{i j}(t) \geq 0$,

(v) $\sum_{j \geq 0} P_{i j}(t) \leq 1 ; i \geq 0, t \geq 0$,

(vi) $\mathscr{P}(t+s)=\mathscr{P}(t) \mathscr{P}(s)$,

where $\mathscr{P}(t)=\left(P_{i j}(t)\right)_{i, j \in \mathbb{N}}$ is the transition matrix. 
4 2-orthogonal polynomials and birth and death processes

Now, we will seek a solution of the Chapman-Kolmogorov equations, by using the method of separation of variables. So if we put

$$
P_{i j}(t)=f(t) F_{i} Q_{j}
$$

we will get the following lemma.

Lemma 2.4. If the transition probabilities satisfy (1.2), then

$$
\begin{gathered}
f(t)=\exp (-x t), \\
F_{0}(x)=1 ; \quad F_{-2}(x) \equiv F_{-1}(x) \equiv 0, \\
-x F_{i}(x)=\lambda_{i} F_{i+1}(x)+\mu_{i} F_{i-1}(x)+\bar{\mu}_{i} F_{i-2}(x) \\
-\left(\bar{\mu}_{i}+\mu_{i}+\lambda_{i}\right) F_{i}(x), \quad i \geq 0, \\
Q_{-1}(x) \equiv 0 ; \quad Q_{0}(x)=1 ; \quad Q_{1}(x)=-\frac{\mu_{0}}{\bar{\mu}_{1}} ; \\
-x Q_{j}(x)=\bar{\mu}_{j+2} Q_{j+2}(x)+\mu_{j+1} Q_{j+1}(x)+\lambda_{j-1} Q_{j-1}(x) \\
-\left(\bar{\mu}_{j}+\mu_{j}+\lambda_{j}\right) Q_{j}(x), \quad j \geq 0 .
\end{gathered}
$$

Proof. By differentiating with respect to $t$ and taking into account that $F_{i}$ and $Q_{j}$ cannot vanish identically, we get

$$
\begin{gathered}
\frac{f^{\prime}(t)}{f(t)}=\frac{1}{Q_{j}}\left[\bar{\mu}_{j+2} Q_{j+2}+\mu_{j+1} Q_{j+1}+\lambda_{j-1} Q_{j-1}-\left(\lambda_{j}+\mu_{j}+\bar{\mu}_{j}\right) Q_{j}\right]=-x, \\
\frac{f^{\prime}(t)}{f(t)}=\frac{1}{F_{i}}\left[\lambda_{i} F_{i+1}+\mu_{i} F_{i-1}+\bar{\mu}_{i} F_{i-2}-\left(\lambda_{i}+\mu_{i}+\bar{\mu}_{i}\right) F_{i}\right]=-x,
\end{gathered}
$$

where $x$ is a separation constant. As $P_{00}(0)=1$, we get (2.9), (2.10), and (2.11).

Remark 2.5. (a) We have written $F_{i}(x)$ and $Q_{j}(x)$ to exhibit the dependence of $F_{i}$ and $Q_{j}$ on the constant $x$.

(b) It is easy to see that the functions $F_{i}(x)$ and $Q_{j}(x)$ defined, respectively, by the recurrence formulas (2.10) and (2.11) are polynomials. Moreover, let us note that $\operatorname{deg} F_{k}=$ $k$ for all $k \in \mathbb{N}$.

To characterize the sequence of polynomials $\left\{F_{i}(x)\right\}_{i \in \mathbb{N}}$, we will introduce $d$-orthogonality notion.

\section{3. $d$-orthogonality}

Definition 3.1. Let $\left\{B_{n}(x)\right\}_{n \geq 0}$ be a monic sequence of polynomials and $\left\{\mathscr{L}_{n}\right\}_{n \geq 0}$ a sequence of linear forms. $\left\{\mathscr{L}_{n}\right\}_{n \geq 0}$ is called the dual sequence of $\left\{B_{n}(x)\right\}_{n \geq 0}$ if and only if

$$
\mathscr{L}_{n}\left(B_{m}\right)=\left\langle\mathscr{L}_{n}, B_{m}\right\rangle=\delta_{m n} ; \quad n, m \geq 0 .
$$


Definition $3.2[2,9]$. Let $\Gamma=\left(\Gamma^{1}, \Gamma^{2}, \ldots, \Gamma^{d}\right)^{T}$ be the $d$ linear forms $(d \geq 1)$. A sequence of polynomials $\left\{B_{n}(x)\right\}_{n \geq 0}$ is said to be $d$-orthogonal with respect to $\Gamma$ if it fulfills

$$
\begin{gathered}
\Gamma^{\sigma}\left(x^{m} B_{n}\right)=0 ; \quad n \geq m d+\sigma, m \geq 0, \\
\Gamma^{\sigma}\left(x^{m} B_{d m+\sigma-1}\right) \neq 0 ; \quad m \geq 0,1 \leq \sigma \leq d .
\end{gathered}
$$

Proposition $3.3[2,9]$. Let $\left\{B_{n}(x)\right\}_{n \geq 0}$ be a monic sequence of polynomials, then the following statements are equivalent.

(a) The sequence $\left\{B_{n}(x)\right\}_{n \geq 0}$ satisfies a recurrence relation with four terms:

$$
\begin{gathered}
B_{0}(x)=1 ; \quad B_{1}(x)=x-\beta_{0} ; \quad B_{2}(x)=\left(x-\beta_{1}\right) B_{1}(x)-\gamma_{1} ; \\
B_{n+3}(x)=\left(x-\beta_{n+2}\right) B_{n+2}(x)-\gamma_{n+2} B_{n+1}(x)-\delta_{n+1} B_{n}(x), \quad n \geq 0,
\end{gathered}
$$

where $\delta_{n+1} \neq 0$ for all $n \in \mathbb{N}$.

(b) The sequence $\left\{B_{n}(x)\right\}_{n \geq 0}$ is 2-orthogonal with respect to $\Gamma=\left(\Gamma^{1}, \Gamma^{2}\right)^{T}$ or with respect to $\mathscr{L}=\left(\mathscr{L}_{0}, \mathscr{L}_{1}\right)^{T}$, because

$$
\Gamma^{\sigma}=\sum_{k \geq 0}^{\alpha-1} \alpha_{k}^{\sigma} \mathscr{L}_{k}, \quad\left(\alpha_{\sigma-1}^{\sigma} \neq 0\right) \text { equivalently } \mathscr{L}_{k}=\sum_{\sigma=1}^{k} \xi_{\sigma}^{k} \Gamma^{\sigma}, \quad\left(\xi_{k}^{k} \neq 0\right)
$$

(c) For any couple $(n, \sigma), \sigma=0,1$, and $n \geq 0$, there exist two polynomials $\Lambda^{\mu}(n, \sigma), \mu=$ 0,1, such that

$$
\mathscr{L}_{2 n+\sigma}=\Lambda^{0}(n, \sigma) \mathscr{L}_{0}+\Lambda^{1}(n, \sigma) \mathscr{L}_{1} \quad \text { for } \sigma=0,1, n \in \mathbb{N},
$$

and satisfying $\operatorname{deg} \Lambda^{\mu}(n, \mu)=n$ for $\mu=0,1, \operatorname{deg} \Lambda^{0}(n, 1) \leq n$ for $n \geq 0$, and $\operatorname{deg} \Lambda^{1}(n, 0) \leq$ $n-1$ for $n \geq 1$.

Remark 3.4. From this proposition, we deduce that the sequence of polynomials $\left\{F_{n}\right\}_{n \in \mathbb{N}}$ is 2 -orthogonal with respect to $\mathscr{L}=\left(\mathscr{L}_{0}, \mathscr{L}_{1}\right)^{T}$.

\section{Integral representation}

In this section, we try to give an integral representation of the $P_{i j}(t)$, which are the solutions of the Chapman-Kolmogov equations. First, we give the following lemma.

LEMMA 4.1. Let there be a generalized birth and death process, where the transition probabilities are given by (1.2), then the sequence $\left\{F_{n}\right\}_{n \geq 0}$ given by (2.10) satisfies the following properties.

(A) Putting $F_{n}(x)=\sum_{k \geq 0} a_{n, k} x^{k}$ for all $n \geq 0$, then

$$
a_{0,0}=1, \quad a_{n, n}=(-1)^{n} \prod_{i=0}^{n-1} \lambda_{i} ; \quad n \geq 1 .
$$


6 2-orthogonal polynomials and birth and death processes

The moments of the two orthogonality forms $c_{n}^{\sigma}=\mathscr{L}_{\sigma}\left(x^{n}\right), n \geq 0$, and $\sigma=0,1$ are given by

$$
\begin{gathered}
c_{0}^{0}=1, \quad c_{0}^{1}=\lambda_{0}+\mu_{0}+\bar{\mu}_{0}, \quad c_{n+2}^{0}=\frac{1}{a_{n+2, n+2}} \sum_{i=0}^{n+1} a_{n+2, i} c_{i}^{0}, \quad n \geq 0 . \\
c_{0}^{1}=0, \quad c_{1}^{1}=-\lambda_{0}, \quad c_{n+2}^{1}=\frac{1}{a_{n+2, n+2}} \sum_{i=0}^{n+1} a_{n+2, i} c_{i}^{1}, \quad n \geq 0 .
\end{gathered}
$$

(B) The following equation holds:

$$
e^{-x t} F_{n}(x)=\sum_{j \geq 0} P_{n j}(t) F_{j}(x), \quad x \in \mathbb{R}, n \in \mathbb{N} .
$$

(C) The sequence $\left\{F_{n}(0)\right\}_{n \geq 0}$ is a nondecreasing sequence and $F_{n}(0) \geq 1(n \geq 0)$.

Proof. (A) If we put $F_{n}(x)=\sum_{k=0}^{n} a_{n, k} x^{k}, n \geq 0$, then from (2.10) we will deduce that

$$
a_{n+1, n+1}=-\frac{1}{\lambda_{n}} a_{n, n},
$$

where

$$
a_{0,0}=1
$$

So, we get (4.1). From the definition of the dual sequence we find (4.2).

(B) Let $\left\{f_{n}\right\}_{n \in \mathbb{N}}$ be a sequence of functions defined by

$$
f_{n}(x, t)=\sum_{j \geq 0} P_{n j}(t) F_{j}(x), \quad n \in \mathbb{N},
$$

or $f(x, t)=\mathscr{P}(t) \mathscr{F}(x)$, where $f(x, t)=\left(f_{n}(x, t)\right)_{n \in \mathbb{N}}$ and $\mathscr{F}(x)=\left(F_{n}(x)\right)_{n \in \mathbb{N}}$, then

$$
\frac{\partial}{\partial t} f(x, t)=\mathscr{P}^{\prime}(t) \mathscr{F}(x)=\mathscr{P}(t) \mathscr{A} \mathscr{F}(x)
$$

From $(2.10)$, we have $-x \mathscr{F}(x)=\mathscr{A} \mathscr{F}(x)$. So,

$$
\begin{gathered}
\frac{\partial}{\partial t} f_{n}(x, t)=-x f_{n}(x, t), \\
f_{n}(x, 0)=F_{n}(x), \quad n \in \mathbb{N} .
\end{gathered}
$$

Then, we get (4.3).

(C) We show by induction that $\left\{F_{n}(0)\right\}_{n \geq 0}$ is a nondecreasing sequence.

Indeed, we have $F_{1}(0)=1+\left(\mu_{0}+\bar{\mu}_{0}\right) / \lambda_{0} \geq F_{0}(0)$.

Assume that $F_{n+1}(0) \geq F_{n}(0) \geq 1$ for all $0 \leq n \leq k-1$, then from (2.10) we get

$$
\lambda_{k}\left(F_{k+1}(0)-F_{k}(0)\right)=\mu_{k}\left(F_{k}(0)-F_{k-1}(0)\right)+\bar{\mu}_{k}\left(F_{k}(0)-F_{k-2}(0)\right) \geq 0 .
$$


So,

$$
F_{n+1}(0) \geq F_{n}(0) \geq 1 ; \quad n \geq 0 .
$$

Theorem 4.2 [1, Boas's theorem]. Let $\left\{\alpha_{n}\right\}_{n \geq 0}$ be a real sequence, then there exists a bounded variation function $\alpha(x)$ defined on $[0,+\infty[$ such that

$$
\int_{0}^{+\infty} x^{n} d \alpha(x)=\alpha_{n}, \quad n \in \mathbb{N}
$$

Remark 4.3. For any element $\mathscr{L}_{m}$ of the dual sequence, there exists a bounded variation function $\Psi_{m}$, such that

$$
\mathscr{L}_{m}\left(x^{n}\right)=\int_{0}^{+\infty} x^{n} d \Psi_{m} ; \quad n, m \in \mathbb{N}
$$

Theorem 4.4. Let $\mathscr{P}(t)=\left(P_{i j}(t)\right)_{i, j \in \mathbb{N}}$ be a solution of (1.3), (2.1), and (2.2), then if

$$
\sum_{j=0}^{m}\left|F_{j}(x)\right| \leq g_{\sigma}(x) \quad \forall x \in \mathbb{R}_{+}, \forall m \in \mathbb{N}, \sigma=0,1,
$$

where $g_{\sigma}(\sigma=0,1)$ is an integrable function with respect to $\Psi_{\sigma}$, the following integral representation holds:

$$
P_{m n}(t)=\int_{0}^{+\infty} e^{-x t} F_{m}(x) d \Psi_{n} \quad n, m \in \mathbb{N},
$$

where $\Psi_{n}, n \geq 0$ is the bounded variation function given in Remark 4.3.

Proof. From (4.3), we deduce that $\left\{P_{n i}(t) F_{i}(x)\right\}_{i \geq 0}$ is a bounded sequence for all $n \in \mathbb{N}$ and $x, t \geq 0$. In particular, there exists a sequence of functions $M_{n}(t)$ such that

$$
\left|P_{n i}(t)\right| \leq \frac{M_{n}(t)}{F_{n}(0)} \leq M_{n}(t) \quad \forall i \in \mathbb{N},
$$

then we have

$$
\left|\sum_{i=0}^{m} P_{n i}(t) F_{i}(x)\right| \leq \sum_{i=0}^{m}\left|P_{n i}(t) F_{i}(x)\right| \leq M_{n}(t) \sum_{i=0}^{m}\left|F_{i}(x)\right| .
$$


8 2-orthogonal polynomials and birth and death processes

Hence $\left\{\sum_{i=0}^{m} P_{n i}(t) F_{i}(x)\right\}_{m \geq 0}$ is dominated by an integrable function with respect to $\Psi_{\sigma}(\sigma=0,1)$. Then, we can write

$$
\begin{aligned}
\int_{0}^{+\infty} e^{-x t} F_{m} d \Psi_{n}(x) & =\sum_{j \geq 0} P_{m j}(t) \int_{0}^{+\infty} F_{j} d \Psi_{n}(x) \\
& =P_{m n}(t) \int_{0}^{+\infty} F_{n} d \Psi_{n}(x)=P_{m n}(t) .
\end{aligned}
$$

Corollary 4.5. Let $\mathscr{P}(t)=\left(P_{i j}(t)\right)_{i, j \in \mathbb{N}}$ be a solution of (1.3), (2.1), and (2.2). Then the $P_{i j}(t)$ given by relation (4.14) can be written in the following form:

$$
\begin{gathered}
P_{n \sigma}(t)=\int_{0}^{+\infty} e^{-x t} F_{n}(x) d \Psi_{\sigma}(x) ; \quad n \geq 0, \sigma=0,1, \\
P_{n, 2 k+\sigma}(t)=\frac{1}{\prod_{j=1}^{k} \bar{\mu}_{2 j+\sigma}}\left[\int_{0}^{+\infty}(-x)^{k} e^{-x t} F_{n}(x) d \Psi_{\sigma}(x)\right. \\
\left.\quad-\sum_{j=0}^{2 k+\sigma-1} P_{n j}(t) \int_{0}^{+\infty}(-x)^{k} F_{j}(x) d \Psi_{\sigma}(x)\right] ; \quad k \geq 0, n \geq 0, \sigma=0,1 .
\end{gathered}
$$

Proof. We have for $t \geq 0$ and $\sigma=0,1$

$$
\begin{aligned}
\int_{0}^{+\infty}(-x)^{m} e^{-x t} F_{n}(x) d \Psi_{\sigma}(x)= & \sum_{j \geq 0} P_{n, 2 j}(t) \int_{0}^{+\infty}(-x)^{m} P_{n j}(t) F_{j}(x) d \Psi_{\sigma}(x) \\
& +\sum_{j \geq 0} P_{n, 2 j+1}(t) \int_{0}^{+\infty}(-x)^{m} P_{n j}(t) F_{j}(x) d \Psi_{\sigma}(x),
\end{aligned}
$$

taking into account that

$$
\begin{aligned}
\mathscr{L}_{0}\left((-x)^{m} F_{2 j+1}(x)\right)=0, & j \geq m, \\
\mathscr{L}_{0}\left((-x)^{m} F_{2 j}(x)\right)=0, & j \geq m+1, \\
\mathscr{L}_{1}\left((-x)^{m} F_{2 j+1}(x)\right)=0, & j \geq m+1, \\
\mathscr{L}_{1}\left((-x)^{m} F_{2 j}(x)\right)=0, & j \geq m+1 .
\end{aligned}
$$

It follows that

$$
\begin{aligned}
& \sum_{j=0}^{m} P_{n, 2 j}(t) \mathscr{L}_{0}\left((-x)^{m} F_{2 j}(x)\right)+\sum_{j=0}^{m-1} P_{n, 2 j+1}(t) \mathscr{L}_{0}\left((-x)^{m} F_{2 j+1}(x)\right)=\mathscr{L}_{0}\left((-x)^{m} e^{-x t} F_{n}\right), \\
& \sum_{j=0}^{m} P_{n, 2 j}(t) \mathscr{L}_{1}\left((-x)^{m} F_{2 j}(x)\right)+\sum_{j=0}^{m} P_{n, 2 j+1}(t) \mathscr{L}_{1}\left((-x)^{m} F_{2 j+1}(x)\right)=\mathscr{L}_{1}\left((-x)^{m} e^{-x t} F_{n}\right),
\end{aligned}
$$


for all $n$ and $m \in \mathbb{N}$, which is a system with lower triangular matrix of the form $\mathscr{P}_{m} \mathscr{P}_{n}(t)=$ $Q_{n m}$, where $\mathscr{B}_{m}=\left(b_{i, j}\right)_{i, j=0}^{2 m+1}$ and $Q_{n m}=\left(q_{n, i}\right)_{i=0}^{2 m+1}$ such that

$$
\begin{gathered}
b_{\sigma, \sigma}=\mathscr{L}_{\sigma}\left(F_{\sigma}\right)=1, \quad b_{10}=b_{01}=0, \quad \sigma=0,1, \\
b_{2 i+\sigma, j}=\mathscr{L}_{\sigma}\left((-x)^{i} F_{j}\right), \quad i=\overline{0, m} ; j=\overline{0,2 i+\sigma} \sigma=0,1, \\
q_{n, 2 i+\sigma}=q_{n 2 i+\sigma}(t)=\mathscr{L}_{\sigma}\left((-x)^{i} e^{-x t} F_{n}\right) \quad i=\overline{0, m} \sigma=0,1,
\end{gathered}
$$

where the determinant of $\mathscr{B}_{m}$ is

$$
\Delta_{m}=\prod_{j=1}^{m} \mathscr{L}_{0}\left((-x)^{j} F_{2 j}\right) \mathscr{L}_{1}\left((-x)^{j} F_{2 j+1}\right)>0,
$$

because

$$
\mathscr{L}_{\sigma}\left((-x)^{j} F_{2 j}\right)=\prod_{i=1}^{j} \bar{\mu}_{2 i+\sigma}>0, \quad j \geq 1 \sigma=0,1 .
$$

So, this system admits a unique solution given by (4.18) for all $n \geq 0$ and $\sigma=0,1$.

Now, we give the sufficient conditions so that the $P_{i j}(t)$ given by the integral representation (4.14) is indeed a transition probability.

First, we give the following lemma.

Lemma 4.6. Let there be a system with a lower triangular matrix of the form $M_{n} X_{n}=C_{n}$, where

$$
\begin{gathered}
M_{n}=\left(\begin{array}{ccccc}
m_{00} & 0 & 0 & \cdots & 0 \\
m_{10} & m_{11} & 0 & \cdots & 0 \\
m_{20} & m_{21} & m_{22} & \cdots & 0 \\
\vdots & \vdots & \vdots & \ddots & \vdots \\
m_{n 0} & m_{n 1} & m_{n 2} & \cdots & m_{n n}
\end{array}\right), \\
X_{n}=\left(x_{i}\right)_{i=0}^{n}, \quad C_{n}=\left(c_{i}\right)_{i=0}^{n} .
\end{gathered}
$$

Then the following properties are equivalent.

(A) $\sum_{i=0}^{n} x_{i} \leq 1$.

(B) $\operatorname{det} D_{n}$ and $\operatorname{det} M_{n}$ have the same sign, where $D_{n}$ is the matrix defined by

$$
D_{n}=\left(\begin{array}{cccccc}
1 & 1 & 1 & 1 & \cdots & 1 \\
c_{0} & m_{00} & 0 & 0 & \cdots & 0 \\
c_{1} & m_{10} & m_{11} & 0 & \cdots & 0 \\
c_{2} & m_{20} & m_{21} & m_{22} & \cdots & 0 \\
\vdots & \vdots & \vdots & \vdots & \ddots & \vdots \\
c_{n} & m_{n 0} & m_{n 1} & m_{n 2} & \cdots & m_{n n}
\end{array}\right) .
$$


Proof. Using Cramer's rule, on one hand we can get

$$
\begin{aligned}
& x_{i}=\frac{1}{\operatorname{det} M_{n}}\left|\begin{array}{cccccccc}
m_{00} & 0 & \cdots & 0 & c_{0} & \cdots & 0 & 0 \\
m_{10} & m_{11} & \cdots & 0 & c_{1} & \cdots & 0 & 0 \\
\vdots & \vdots & \ddots & \vdots & \vdots & \vdots & \vdots & \vdots \\
m_{i-10} & m_{i-11} & \cdots & m_{i-1 i-1} & c_{i-1} & \cdots & 0 & 0 \\
m_{i 0} & m_{i 1} & \cdots & m_{i i-1} & c_{i} & \cdots & 0 & 0 \\
\vdots & \vdots & \vdots & \vdots & \vdots & \ddots & \vdots & \vdots \\
m_{n-10} & m_{n-11} & \cdots & m_{n-1 i-1} & c_{n-1} & \cdots & m_{n-1 n-1} & 0 \\
m_{n 0} & m_{n 1} & \cdots & m_{n i-1} & c_{n} & \cdots & \cdots m_{n n-1} & m_{n n}
\end{array}\right| \\
& =\frac{(-1)^{i}}{\operatorname{det} M_{n}}\left|\begin{array}{cccccccc}
c_{0} & m_{00} & 0 & \cdots & 0 & \cdots & 0 & 0 \\
c_{1} & m_{10} & m_{11} & \cdots & 0 & \cdots & 0 & 0 \\
\vdots & \vdots & \ddots & \vdots & \vdots & \vdots & \vdots & \vdots \\
c_{i-1} & m_{i-10} & m_{i-11} & \ddots & m_{i-1 i-1} & \cdots & 0 & 0 \\
c_{i} & m_{i 0} & m_{i 1} & \cdots & m_{i i-1} & \cdots & 0 & 0 \\
\vdots & \vdots & \vdots & \vdots & \vdots & \ddots & \vdots & \vdots \\
c_{n-1} & m_{n-10} & m_{n-11} & \cdots & m_{n-1 i-1} & \cdots & m_{n-1 n-1} & 0 \\
c_{n} & m_{n 0} & m_{n 1} & \cdots & m_{n i-1} & \cdots & m_{n n-1} & m_{n n}
\end{array}\right|,
\end{aligned}
$$

and on the other hand, we have

$$
\begin{aligned}
& \operatorname{det} D_{n}=\operatorname{det} M_{n} \\
& -\sum_{i=0}^{n}(-1)^{i}\left|\begin{array}{cccccccc}
c_{0} & m_{00} & 0 & \ldots & 0 & \cdots & 0 & 0 \\
c_{1} & m_{10} & m_{11} & \ldots & 0 & \cdots & 0 & 0 \\
\vdots & \vdots & \ddots & \vdots & \vdots & \vdots & \vdots & \vdots \\
c_{i-1} & m_{i-10} & m_{i-11} & \ddots & m_{i-1 i-1} & \cdots & 0 & 0 \\
c_{i} & m_{i 0} & m_{i 1} & \ldots & m_{i i-1} & \cdots & 0 & 0 \\
\vdots & \vdots & \vdots & \vdots & \vdots & \ddots & \vdots & \vdots \\
c_{n-1} & m_{n-10} & m_{n-11} & \ldots & m_{n-1 i-1} & \cdots & m_{n-1 n-1} & 0 \\
c_{n} & m_{n 0} & m_{n 1} & \ldots & m_{n i-1} & \cdots & m_{n n-1} & m_{n n}
\end{array}\right| .
\end{aligned}
$$

So, $\operatorname{det} D_{n}=\operatorname{det} M_{n}\left(1-\sum_{i=0}^{n} x_{i}\right)$ with $\operatorname{det} M_{n} \neq 0$, consequently we deduce that (A) and (B) are equivalent.

From Corollary 4.5 and Lemma 4.1, we have the following result.

Theorem 4.7. Let $\mathscr{P}(t)=\left(P_{i j}(t)\right)_{i, j \in \mathbb{N}}$ be a solution of (1.3), (2.1), and (2.2). If the following conditions are satisfied:

(i) $\int_{0}^{+\infty} e^{-x t} F_{n}(x) d \Psi_{\sigma} \geq 0$, for all $n \in \mathbb{N}$ and $\sigma=0,1$, 
(ii) $\operatorname{det} \mathscr{D}_{n m} \geq 0$ for all $n, m \in \mathbb{N}$, where $\mathscr{D}_{n m}$ is the following matrix:

$$
D_{n m}=\left(\begin{array}{cccccc}
1 & 1 & 1 & 1 & \cdots & 1 \\
q_{n 0} & b_{00} & 0 & 0 & \cdots & 0 \\
q_{n 1} & b_{10} & b_{11} & 0 & \cdots & 0 \\
q_{n 2} & b_{20} & b_{21} & b_{22} & \cdots & 0 \\
\vdots & \vdots & \vdots & \vdots & \ddots & \vdots \\
q_{n 2 m+1} & b_{2 m+10} & b_{2 m+11} & b_{2 m+12} & \cdots & b_{2 m+12 m+1}
\end{array}\right)
$$

where $q_{n j}$ and $b_{i j}$ are given by (4.22),

(iii)

$$
\int_{0}^{+\infty}(-x)^{k} F_{j}(x) d \Psi_{\sigma} \leq \int_{0}^{+\infty}(-x)^{k} e^{-x t} F_{n}(x) d \Psi_{\sigma} ; \quad j=\overline{0,2 k+\sigma-1}
$$

for all $n \in \mathbb{N}, k \in \mathbb{N}^{*}$ and $t>0$, then

$$
P_{n m}(t) \geq 0 ; \quad n, m \geq 0, \quad \sum_{i=0}^{m} P_{n i}(t) \leq 1 \quad \forall n, m \in \mathbb{N}, t \geq 0 .
$$

Proof. If condition (ii) is satisfied, we deduce from Lemma 4.1 that

$$
\sum_{i=0}^{m} P_{n i}(t) \leq 1 \quad \forall n, m \in \mathbb{N}, t \geq 0
$$

From (4.14), we can get $P_{n m}(0)=\delta_{n m}$.

As $(t>0)$

$$
\begin{aligned}
P_{n, 2 k+\sigma}(t)=\frac{1}{\prod_{j=1}^{k} \bar{\mu}_{2 j+\sigma}} & {\left[\int_{0}^{+\infty}(-x)^{k} e^{-x t} F_{n}(x) d \Psi_{\sigma}\right.} \\
& \left.-\sum_{j=0}^{2 k+\sigma-1} P_{n j}(t) \int_{0}^{+\infty}(-x)^{k} F_{j}(x) d \Psi_{\sigma}\right], \quad k \geq 1,
\end{aligned}
$$

then $P_{n, 2 k+\sigma}(t) \geq 0$, that is,

$$
\int_{0}^{+\infty}(-x)^{k} e^{-x t} F_{n}(x) d \Psi_{\sigma} \geq \sum_{j=0}^{2 k+\sigma-1} P_{n j}(t) \int_{0}^{+\infty}(-x)^{k} F_{j}(x) d \Psi_{\sigma} \quad \forall k \geq 1 .
$$

We will show by induction on $m$ that $P_{n m}(t) \geq 0$ for all $n \geq 0$. From (i) we have $P_{n, 0}(t) \geq 0$ and $P_{n 1}(t) \geq 0$.

Assume that

$$
P_{n m}(t) \geq 0 ; \quad 0 \leq m \leq j .
$$


For any $j$, there exists $k$ such that $j=2 k+\sigma-1$, it follows that

$$
\begin{aligned}
\sum_{m=0}^{2 k+\sigma-1} P_{n m}(t) \int_{0}^{+\infty}(-x)^{k} F_{m}(x) d \Psi_{\sigma} & \leq \max _{0 \leq m \leq 2 k+\sigma-1}\left\{\int_{0}^{+\infty}(-x)^{k} F_{m}(x) d \Psi_{\sigma}\right\}^{2 k+\sigma-1} \sum_{m=0} P_{n m}(t) \\
& \leq \max _{0 \leq m \leq 2 k+\sigma-1}\left\{\int_{0}^{+\infty}(-x)^{k} F_{m}(x) d \Psi_{\sigma}\right\} .
\end{aligned}
$$

Since (iii) is satisfied, we obtain $P_{n, j+1}(t) \geq 0$.

Hence, we get $P_{n m}(t) \geq 0$ for all $n$ and $m \geq 0$.

\section{References}

[1] R. P. Boas Jr., The Stieltjes moment problem for functions of bounded variation, Bulletin of the American Mathematical Society 45 (1939), 399-404.

[2] K. Douak and P. Maroni, Une caractérisation des polynômes d-orthogonaux "classiques" [A characterization of "classical" d-orthogonal polynomials], Journal of Approximation Theory 82 (1995), no. 2, 177-204.

[3] M. E. H. Ismail, J. Letessier, and G. Valent, Linear birth and death models and associated Laguerre polynomials, Journal of Approximation Theory 56 (1988), no. 3, 337-348.

[4] Luadratic birth and death processes and associated continuous dual Hahn polynomials, SIAM Journal on Mathematical Analysis 20 (1989), no. 3, 727-737.

[5] M. E. H. Ismail, D. R. Masson, J. Letessier, and G. Valent, Birth and death processes and orthogonal polynomials, Orthogonal Polynomials and Their Applications (Columbus, Ohio, 1989) (P. Nevai, ed.), NATO Adv. Sci. Inst. Ser. C Math. Phys. Sci., vol. 294, Kluwer Academic, Dordrecht, 1990, pp. 229-255.

[6] S. Karlin and J. McGregor, The differential equations of birth-and-death processes, and the Stieltjes moment problem, Transactions of the American Mathematical Society 85 (1957), no. 2, 489546.

[7] J. Letessier and G. Valent, The generating function method for quadratic asymptotically symmetric birth and death processes, SIAM Journal on Applied Mathematics 44 (1984), no. 4, 773-783.

[8] D. P. Maki, On birth-death processes with rational growth rates, SIAM Journal on Mathematical Analysis 7 (1976), no. 1, 29-36.

[9] P. Maroni, L'orthogonalité et les récurrences de polynômes d'ordre supérieur à deux, Annales de la Faculté des Sciences de Toulouse. Série VI. Mathématiques 10 (1989), no. 1, 105-139.

Zerouki Ebtissem: Department of Mathematics, Faculty of Science, University of Badji Mokhtar, Annaba, P.O. Box 12, Annaba 23000, Algeria

E-mail address: ebzerouki@yahoo.fr

Boukhemis Ammar: Department of Mathematics, Faculty of Science, University of Badji Mokhtar, Annaba, P.O. Box 12, Annaba 23000, Algeria

E-mail address: aboukhemis@yahoo.com 


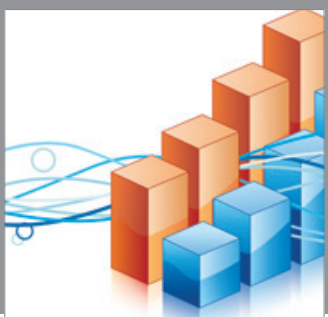

Advances in

Operations Research

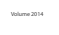

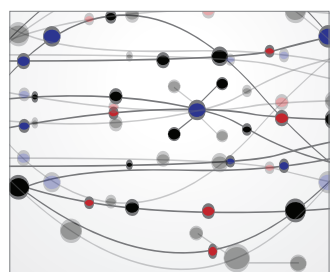

\section{The Scientific} World Journal
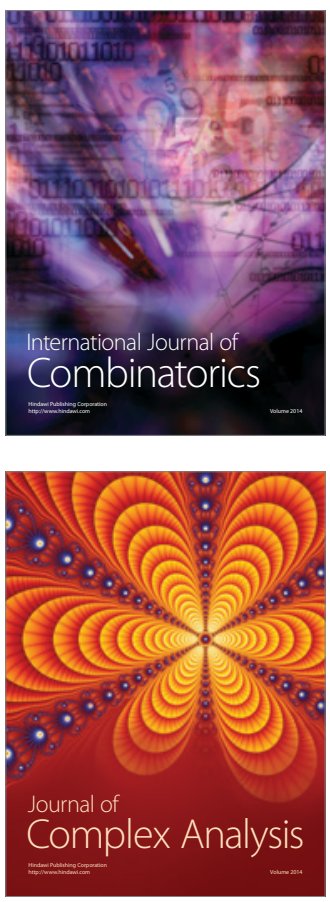

International Journal of

Mathematics and

Mathematical

Sciences
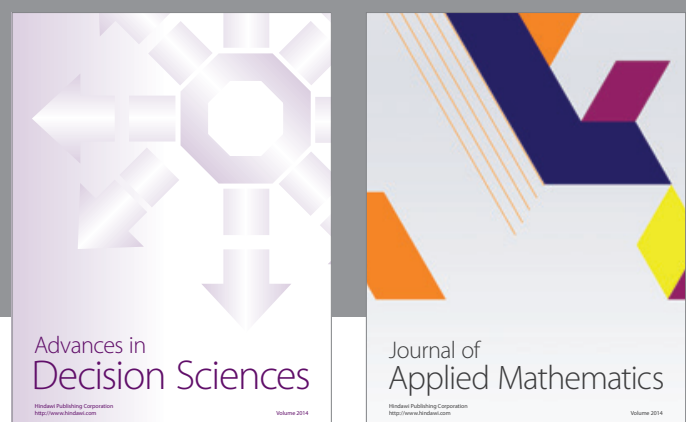

Journal of

Applied Mathematics
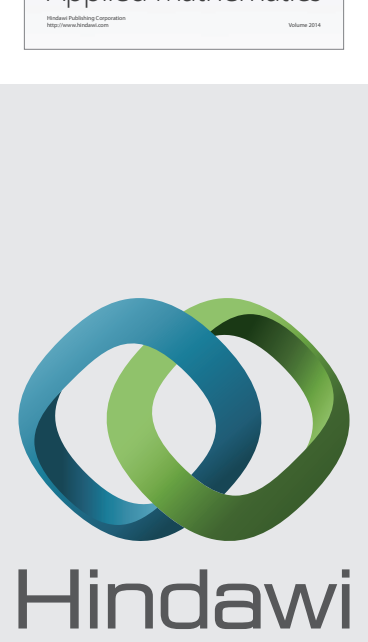

Submit your manuscripts at http://www.hindawi.com
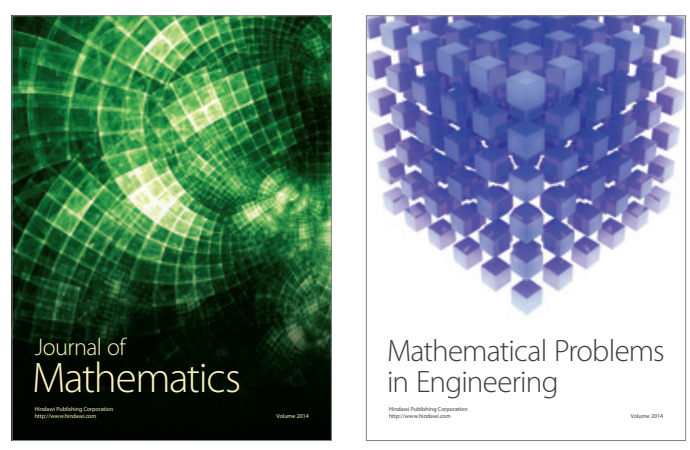

Mathematical Problems in Engineering
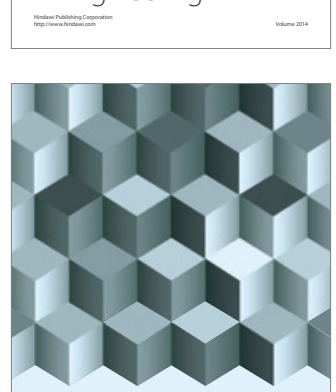

Journal of

Function Spaces
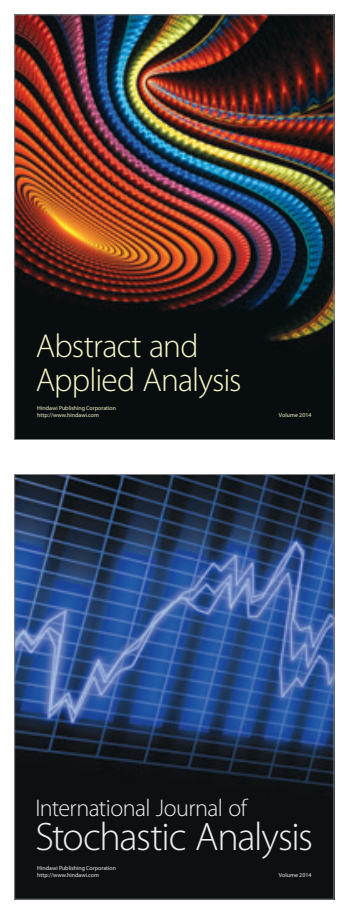

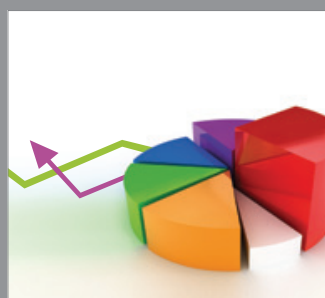

ournal of

Probability and Statistics

Promensencen
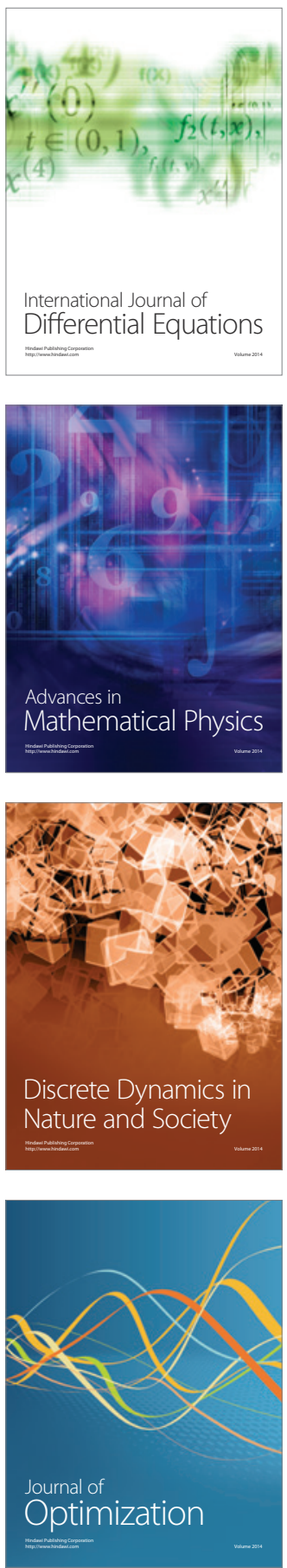